\title{
Discovery of a novel tetrapeptide against Influenza A virus: ra- tional design, synthesis, bioactivity evaluation and computa- tional studies
}

\author{
Maria Carmina Scala1 ${ }^{1,}$, Mariangela Agamennone ${ }^{2, \$},{\text { Agostina Pietrantoni }{ }^{3,4}, \text { Veronica Di Sarno }}^{1}$, Alessia \\ Bertamino $^{1}$, Fabiana Superti ${ }^{3}$, Pietro Campiglia ${ }^{1}$ and Marina Sala ${ }^{1}{ }^{*}$ \\ 1 Department of Pharmacy, University of Salerno, Via Giovanni Paolo II 132, 84084, Fisciano (SA), Italy; \\ mscala@unisa.it (M.C.S.); vdisarno@unisa.it (V.D.); abertamino@unisa.it (A.B.); pcampiglia@unisa.it (P.C.) \\ 2 Department of Pharmacy, University “G. d'Annunzio" of Chieti-Pescara, Via dei Vestini 31, 66100, Chieti, \\ Italy; magamennone@unich.it (M.A.) \\ 3 National Centre for Innovative Technologies in Public Health, National Institute of Health, Viale Regina \\ Elena 299, 00161, Rome, Italy; agostina.pietrantoni@iss.it (A.P); fabiana.superti@iss.it (F.S.) \\ 4 Core Facilities, National Institute of Health, Viale Regina Elena 299, 00161, Rome, Italy (A.P.) \\ * Correspondence: msala@unisa.it; Tel.: +39-(0)89968148 \\ $\S$ These authors contributed equally to this work
}

\begin{abstract}
Influenza is a highly contagious, acute respiratory illness, which represents one of the main health issues worldwide. Even though some antivirals are available, the alarming increase of virus strains resistant to them highlights the need to find new drugs. Previously, Superti et al. have deeper investigated the mechanism of the anti-Influenza virus effect of bovine Lactoferrin (bLf) and the role of its tryptic fragments (the $\mathrm{N}$ and C-lobes) in the antiviral activity. Recently, through a truncation library, we identified the tetrapeptides, SKHS (1) and SLDC (2), derived from bLf C-lobe fragment 418-429, which were able to bind hemagglutinin (HA) and inhibit cell infection in a concentration range of femto- to picomolar. Starting from these results, in this work, we initiated a systematic SAR study on the peptides mentioned above, through an Alanine scanning approach. We carried out binding affinity measurements by microscale thermophoresis (MST) and Surface Plasmon Resonance (SPR), and hemagglutination inhibition (HI) and virus neutralization (NT) assays on synthesized peptides. Computational studies were performed to identify possible ligandHA interactions. Results obtained led to the identification of an interesting peptide endowed with broad anti-Influenza activity and able to inhibit viral infection to a greater extent of reference peptide.
\end{abstract}

Keywords: Influenza A virus; lactoferrin; tetrapeptides; biophysics; antiviral agents; hemagglutinin

\section{Introduction}

Influenza is a highly contagious, acute respiratory illness, which represents one of the most important health issue worldwide. There are three types of Influenza viruses that infect humans: A, B and C. Influenza A viruses (IAV) also naturally infect a variety of other animal species and are the only Influenza viruses known to cause influenza pandemics, which are global epidemics of influenza diseases [1]. The recent outbreak of Sars-CoV-2 dramatically demonstrated the risks of a global viral spread and highlighted the key role of prevention. In this context, Influenza A virus is a highly monitored pathogen: it is largely diffused in the avian population and its spillover to humans could 
represent a serious threat [2]. Despite its global diffusion, just a few drugs are on the clinic, with vaccination representing the main strategy for preventing infections. Efforts to influenza prevention by vaccination are made difficult by the virus ability to rapidly mutate and recombine into antigenically new viral particles, sometimes leading to the emergence of a totally new viral strain. For this reason, at present, the development of antiviral drugs represents a crucial strategy in the control and prevention of seasonal and pandemic influenza infections [3]. Three classes of antiviral drugs have been approved for treatment and prophylaxis of influenza [4] the adamantane derivatives (amantadine and rimantadine), potent M2 channel blockers [5], neuraminidase inhibitors (NAIs: zanamivir, oseltamivir laninamivir and peramivir) $[6,7]$, and the selective inhibitor of Influenza capdependent endonuclease (baloxavir marboxil) [8].

However, the capability of viruses to mutate the target proteins represents an obstacle to efficient treatment with these drugs. Based on the above considerations it is evident the need for new compounds against Influenza virus able to overcome the disadvantages of the known therapies [9, 10].

An attractive antiviral strategy is the blocking of Influenza virus entry into the host cell. This process is mediated by viral hemagglutinin. HA is the major surface protein of IAV and is essential to the entry process so representing an attractive target for antiviral therapy. It is a large homotrimeric mushroom-shaped glycoprotein responsible for the initial attachment to the host cell through the receptor binding site (RBS), and the successive viral internalization through the membrane fusion promoted by the structural rearrangement of the conserved stem region of HA (Fig. 1). As a matter of fact, neutralizing compounds targeting HA represent a useful tool in neutralizing viral infection. One notable difficulty in targeting HA is related to its sequence variability among different strains (18 HAs have been identified so far).

Previously, Superti et al. have deeper investigated the mechanism of the antiInfluenza virus effect of bLf and the role of its tryptic fragments (the $\mathrm{N}$ and C-lobes) in the antiviral activity [11]. In particular, they evaluated the influence of bLf on hemagglutininmediated functions [12].

Recently, through a truncation library, we identified the tetrapeptides, SKHS (1) and SLDC (2), derived from bLf C-lobe fragment 418-429, which were able to bind HA and inhibit cell infection [13]. With this tool in hand, in this work, we further investigated the role of key residues of both peptides, in the interaction with HA to better define the influence of the chemical modifications introduced in the peptides on their biological properties in terms of affinity and activity. Direct binding assays, bioactivity profile and computational studies brought to the identification of a very potent and broad spectrum tetrapeptide. 


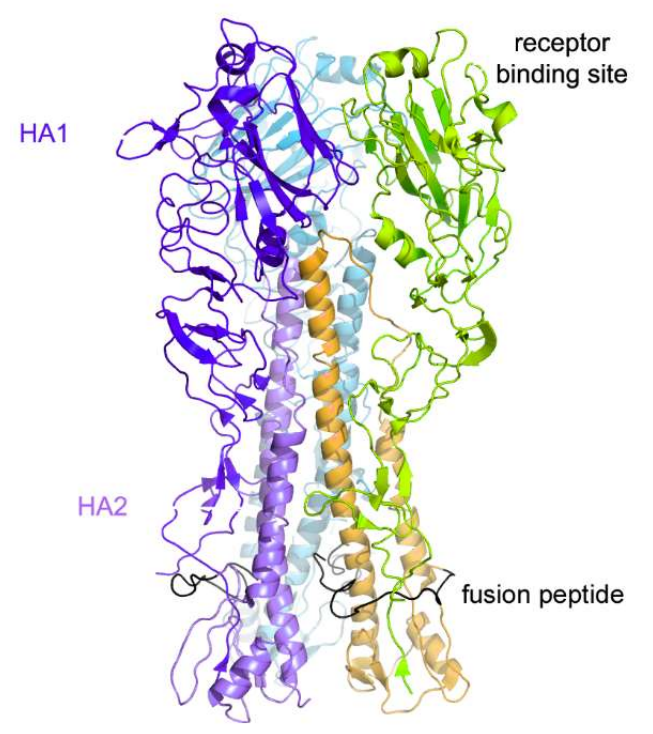

Figure 1. Cartoon representation of the HA trimeric structure.

\section{Results}

\subsection{Design and Synthesis}

The contribution of the various amino acid residues to peptides 1 and 2 activity was further established through an L-Ala scanning analysis [14]. This approach allows to determine the contribution of side chains of each amino acid residue in the interaction with the target molecule, hemagglutinin. This approach resulted in the generation of a panel of eight peptides (peptides 3-10, Table 1).

Peptides were synthesized according to the solid phase approach using standard Fmoc methodology in a manual reaction vessel (Experimental Section). The purification was achieved using a preparative RP-HPLC C-18 bonded silica column. The purified peptides were $98 \%$ pure as determined by analytical RP-HPLC. The correct molecular weight of the peptides was confirmed by mass spectrometry and amino acid analysis (Supplementary material).

\subsection{Direct binding assays}

There are many biophysical methods available to measure the affinity of ligand-protein interactions. Each technique affords a variety of information on the binding specificity, kinetics thermodynamics, stoichiometry, with their advantages and disadvantages, which have been extensively analyzed elsewhere [15]. No single biophysical technique is better than another, as they are based on different principles. For this reason, a more satisfactory approach would be to adopt a strategy that uses one or more orthogonal assays that aim to confirm activity on the target. Based on this consideration, we decided to investigate the direct binding of peptides 1,2 , and the alanine scanning peptide library to HA protein by two complementary techniques: microscale thermophoresis (Fig. 2) and surface plasmon resonance (Fig. 3). 


\subsubsection{Microscale thermophoresis (MST)}

MST is performed using thin capillaries in free solution, which are illuminated with an infrared laser that generates a temperature gradient. The directed movement of molecules is detected by intrinsic fluorescence or in most cases, fluorescent labels of one interactant, and quantified. The thermophoretic movement of molecules within the temperature gradient depends on size, charge, hydration shell, or conformation that typically changes upon interaction. The thermophoresis signal is plotted against the ligand concentration to obtain a dose-response curve, from which the binding affinity can be deduced [16].

MST screening of the 10 compounds was performed as detailed in the Experimental section, and results are reported in Table 1. Analysis revealed that all peptides interact with HA with different dissociation constants, in particular, compound 4 binds HA with higher efficiency with respect to 1 (Fig. 2), showing an equilibrium dissociation constant (KD) value of $8.22 \pm 0.1 \mathrm{nM}$ and $7.26 \pm 0.06 \mu \mathrm{M}$, respectively.
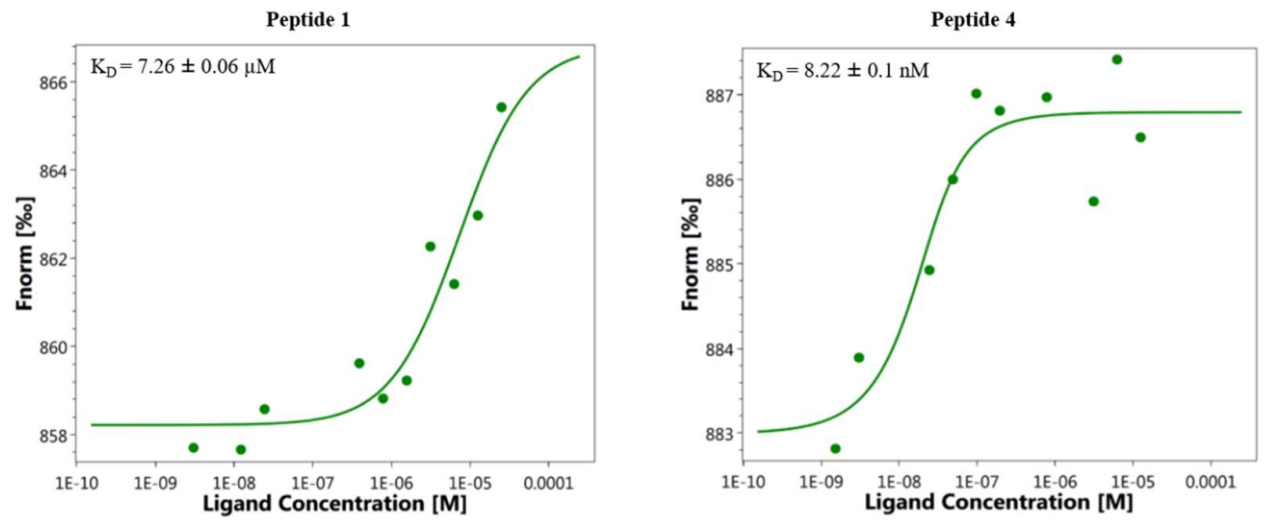

Figure 2. Direct binding measurements of the interaction between HA and peptide $\mathbf{1}$ and 4. MST binding affinity assays were performed as described in Experimental Section. Representative dose-response curves of peptides 1 and $\mathbf{4}$ binding to HA are shown; Fnorm, normalized fluorescence. Experiments were repeated independently three times. Reported $\mathrm{K}_{\mathrm{D}}$ is the mean $\pm \mathrm{SD}$ of three independent experiments.

\subsubsection{Surface Plasmon Resonance (SPR)}

For the SPR study, full-length recombinant HA (His-Tag) was immobilized on a sensor chip up to 12000 response units, RU (Experimental section). Compound binding induced a change in the refractive index on the sensor surface [17]. A regeneration step was necessary (glycine $\mathrm{pH} 1.5$, data not shown). After injection, running buffer was allowed to flow over the surface and the dissociation of compounds from the surface was observed. In contrast, the control flow cell, where no HA was immobilized, showed no significant signal changes (data not shown). The ability of the tetrapeptides to bind HA was defined by their $K_{D}$ values.

SPR analysis showed the synthesized peptides efficiently interacting with the immobilized protein. Figure 3 presents the sensorgrams of compounds 1 and 4 bound to HA in HBS-P buffer. Interestingly, tetrapeptide 4 binds HA with higher efficiency with respect to 1 , showing a $K_{D}$ value of $3.57 \pm 0.12 \mathrm{nM}$ and $4.53 \pm 0.08 \mu \mathrm{M}$, respectively.

Both the direct binding measurements demonstrated specific binding between peptide 4 and HA. The $\mathrm{K}_{\mathrm{D}}$ of the $4: \mathrm{HA}$ complex was $8.22 \pm 0.10 \mathrm{nM}$ in the MST assay and $3.57 \pm 0.12$ $\mathrm{nM}$ in the SPR assay. This is common as the $\mathrm{K}_{\mathrm{D}}$ values deeply depend on the analysis method and the applied setup. 

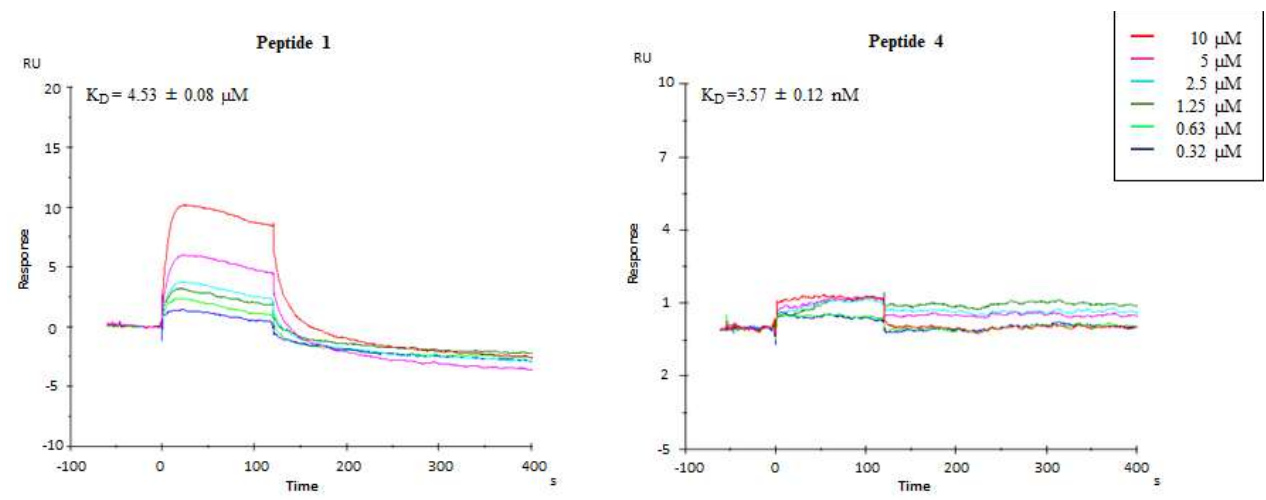

Figure 3. Sensorgrams obtained from the SPR interaction analysis of peptides $\mathbf{1}$ and $\mathbf{4}$ binding to immobilized HA. Each compound was injected at six different concentrations $(0.32,0.63,1.25,2.5,5$, and $10 \mu \mathrm{M})$. The equilibrium dissociation constants $\left(K_{D}\right)$ were derived from the ratio between kinetic dissociation ( $\left.k_{\text {off }}\right)$ and association $\left(k_{o n}\right)$ constants.

\subsection{Antiviral activity}

\subsubsection{Hemagglutination inhibition assay $(\mathrm{HI})$}

The ability of peptides to inhibit the HA activity was assessed by HI. The inhibitory effect of peptides 1 and $\mathbf{2}$ is reported as a reference. The IAV strains A/Roma-ISS/02/08 H1N1 oseltamivir-sensitive virus, A/Parma/24/09 H1N1 oseltamivir-resistant virus, and A/Parma/05/06 H3N2 were used. As shown in Table 1, Ser1, His3, and Ser4 of peptide 1 are key amino acids for the antiviral activity against some of the IAV strains used in the assay. The substitution of Ser1 with an alanine determines the loss of activity against Influenza A/Parma/24/09 H1N1 virus subtype of the corresponding analogue 3 compared to reference peptide 1 . The absence of positive charge by substitution of Lys2 with Ala determines a significant increase of inhibitory potency of the corresponding analogue 4 compared to reference peptide $\mathbf{1}$. Peptide 5 increases the inhibitor potency on Influenza A/H1N1 strains, showing that His3 is important for inhibitory activity of Influenza A/H3N2 virus subtype. In derivative 6 , the substitution of a hydroxyl chain (Ser4) with a more lipophilic residue (Ala) induces a dramatic loss of activity against the two different Influenza $\mathrm{A} / \mathrm{Parma}$ virus subtypes, increasing the antiviral potency on Influenza $\mathrm{A} /$ RomaISS/2/08 A/H1N1 viral strain. The data also showed that the substitution of each amino acidic residue of peptide 2 with an alanine determines the loss of activity against Influenza $\mathrm{A} /$ Roma-ISS/2/08 H1N1 virus subtype, increasing the antiviral potency on the other two different $\mathrm{A} /$ Parma virus subtypes.

Therefore, only one peptide, 4 , was able to prevent HA activity of all tested viral strains. In particular, this peptide exerted a strong antiviral action, in the femtomolar range, against two viral strains: A/Roma-ISS/02/08 H1N1 and A/Parma /05/06 H3N2.

Table 1. Structure Characterization, Affinity, and Activity of peptides 1-10. The HI activity of peptides $\mathbf{1}$ and $\mathbf{2}$ is reported as a reference aScala et al. (2017) [13]). All peptides are amidated and acetylated. 


\begin{tabular}{|c|c|c|c|c|c|c|}
\hline \multirow[b]{2}{*}{ Pep. } & \multirow[b]{2}{*}{ Seq. } & \multirow{2}{*}{$\begin{array}{c}\text { MST KD } \\
(\mu \mathrm{M})\end{array}$} & \multirow{2}{*}{$\begin{array}{c}\text { SPR KD } \\
(\mu \mathrm{M})\end{array}$} & \multicolumn{3}{|c|}{ HI titer (nM) } \\
\hline & & & & $\begin{array}{c}\text { A/Roma- } \\
\text { ISS/02/08 } \\
\text { H1N1 }\end{array}$ & $\begin{array}{c}\text { A/Parma/ } \\
\text { 24/09 } \\
\text { H1N1 } \\
\end{array}$ & $\begin{array}{c}\text { A/Parma/ } \\
\text { 05/06 } \\
\text { H3N2 } \\
\end{array}$ \\
\hline $1^{a}$ & SKHS & $7.26 \pm 0.06$ & $4.53 \pm 0.08$ & 0.1 & 1.5 & 12 \\
\hline 3 & AKHS & $3.12 \pm 0.11$ & $2.7 \pm 0.04$ & 0.6 & - & 12 \\
\hline 4 & SAHS & $0.0082 \pm 0.0001$ & $0.0035 \pm 0.00012$ & $1.8^{*} 10^{-6}$ & 0.5 & $2.4 * 10^{-6}$ \\
\hline 5 & SKAS & $7.01 \pm 0.09$ & $1.03 \pm 0.01$ & $1.8^{*} 10^{-6}$ & $2.9 * 10^{-3}$ & - \\
\hline 6 & SKHA & $11.4 \pm 0.17$ & $6.75 \pm 0.81$ & $5 * 10^{-7}$ & - & - \\
\hline $2^{a}$ & SLDC & $10.4 \pm 0.23$ & $7.12 \pm 0.26$ & $1.4 * 10^{-6}$ & 6 & 1.5 \\
\hline 7 & ALDC & $21.2 \pm 0.41$ & $0.0277 \pm 0.0017$ & - & $9 * 10^{-7}$ & $3.6^{*} 10^{-7}$ \\
\hline 8 & SADC & $6.38 \pm 0.21$ & $2.19 \pm 0.51$ & - & $2.2 * 10^{-6}$ & $5 * 10^{-7}$ \\
\hline 9 & SLAC & $0.0058 \pm 0.0003$ & $2.57 \pm 0.34$ & - & $5 * 10^{-7}$ & $9 * 10^{-7}$ \\
\hline 10 & SLDA & $2.69 \pm 0.09$ & $0.343 \pm 0.019$ & - & 6.1 & $2.1 * 10^{-3}$ \\
\hline
\end{tabular}

\subsubsection{Neutralization assay (NT)}

Prompted by previous findings, we assessed the ability of peptide 4 to affect virus replication in Madin-Darby canine kidney (MDCK) cell line by NT. As shown in Table 2, this peptide was able to prevent infection of all tested viruses in a concentration range from about $0.4 \mathrm{fM}$ to $0.9 \mathrm{pM}$, with a relevant antiviral activity against the oseltamivirresistant $\mathrm{A} / \mathrm{H} 1 \mathrm{~N} 1$ strain with an $\mathrm{EC}_{50}$ value of about $0.4 \mathrm{fM}$ and a very high selectivity index. Notably, this peptide was more active against all flu strains compared not only to reference peptide 1 but also to all peptides we studied, starting from bLf C-lobe to all derived peptides $[11,13]$.

Table 2. In vitro antiviral activity of peptides 1 and $\mathbf{4}$ against Influenza virus infection. aEC50: the reciprocal substance dilution at which $50 \%$ of cells were protected from the virus induced killing; ${ }^{\wedge} \mathrm{SI}$ : the ratio between $\mathrm{CC}_{50}$ (the reciprocal substance dilution at which $50 \%$ of cells were protected from substance toxicity, corresponding to a concentration $>25$ $\mu \mathrm{M})$ and $\mathrm{EC}_{50}$; The mean values of 3 independent experiments with standard errors are shown. bScala et al., (2017) [13]. 


\section{A/Roma-ISS/02/08 H1N1 A/Parma/24/09 H1N1 A/Parma /05/06 H3N2}

\begin{tabular}{cccccccc} 
Pep. Seq. & \multicolumn{1}{c}{$\begin{array}{c}\mathrm{EC}_{50^{\mathrm{a}}} \\
(\mu \mathrm{M})\end{array}$} & SI & $\begin{array}{c}\mathrm{EC}_{50^{\mathrm{a}}} \\
(\mu \mathrm{M})\end{array}$ & SI & $\begin{array}{c}\mathrm{EC}_{50^{\mathrm{a}}} \\
(\mu \mathrm{M})\end{array}$ & SI \\
\hline $\mathbf{1}^{\mathrm{b}}$ & SKHS & $3 \pm 0.61^{*} 10^{-6}$ & $>8.33 .10^{6}$ & $4.8 \pm 0.12^{*} 10^{-8}$ & $>5.2 .10^{8}$ & $5 \pm 0.02^{*} 10^{-6}$ & $>5.10^{6}$ \\
& & & & & & & \\
4 & SAHS & $5.77 \pm 0.01^{*} 10^{-7}$ & $>4.33 .10^{7}$ & $4.3 \pm 0.3^{*} 10^{-10}$ & $>5.81 .10^{10}$ & $9.36 \pm 0.1^{*} 10^{-7}$ & $>2.67 .10^{7}$
\end{tabular}

\subsection{Computational studies}

To get more clues on the tetrapeptide interaction with studied HAs, a structure-based computational analysis was carried out. To be more accurate in the binding prediction, the homology models of our HAs were obtained using the Swiss-Model website on the basis of their nucleotide sequences kindly provided by Dr.ssa Simona Puzelli (Department of Infectious Diseases, ISS, Rome). The obtained model quality was assessed by the QMean and GMQE algorithms (Supplementary material).

The large dimension of this trimeric protein and the great variability between and among strains make the identification of a univocal region interested in the interaction with ligands more complex. To locate the putative binding sites of our ligands on the HA surface, we evaluated what was suggested from experimental data: HI accounts for the interference with the sialic acid recognition, therefore, the binding in the RBS was evaluated. On the other hand, the neutralization activity of tetrapeptides 1 and 4 demonstrates that these molecules interfere with the HA fusion machinery in the HA stem region, and consequently an additional binding site can be envisioned for our peptides on the HA surface. The hypothesized dual binding is not new in fact it was demonstrated for cyclohexyltaurine in complex with H5 HA [18]. Moreover, the selected sites correspond to the three identified by Yusuf et al with ProBis [19].

The RBS is responsible for the first binding to the sialic acid of glycoproteins on the host cell surface [20]. It represents a relatively conserved region in the receptor binding domain (RBD) that, on the contrary, is hypervariable to escape host immunity, for this reason, it can provide a useful tool to fight IAV, also because the displacement of the sialic acid is favored by its low $\mathrm{K}_{\mathrm{D}}$ in the $\mathrm{mM}$ range [18].

A better depiction of the binding site was provided by SiteMap calculation [21] that confirmed the hydrophilic character of this site. Different activities between the two A/H1N1 strains can be due to few modified amino acids all around the site: in fact, A/Roma-ISS/02/08 H1N1 has a slightly more flexible loop surrounding the site because of the insertion of an additional Ala125 (alignment of A/Roma and A/Parma H1N1 sequences is reported in the Supplementary material).

The docking calculations were carried out setting the SP-peptide docking procedure available in Glide [22, 23, 24] aimed to increase the conformational exploration of ligands during the docking process and retrieving up to 100 docked poses. This procedure is particularly useful to have a picture of possible binding modes of very flexible molecules such as peptides. Because of the large number of resulting geometries, these were clustered to select the most reliable binding pose for each ligand, taking into account both docking score and number of retrieved similar conformations.

Docked poses of the most active compound in the three HAs are reported (Fig. 4). A similar binding mode was obtained for the other ligands. The tetrapeptide $\mathbf{4}$ has a 
conserved binding geometry in the three studied HAs, spanning the whole site. RBS residues involved in $\mathrm{H}$-bond interactions with our ligands are the same that get contacts with the sialic acid in most cases; in particular, residues corresponding to Asp174, Glu211, Gln210, Tyr80, and Thr118 (A/Parma H1N1 numbering) are responsible for the binding with 6'-SLN (PDB ID 3UBN [25]). Most involved ligand residues are the two serine forming a network of H-bonds: one of them, in particular, displaces a conserved water molecule with its $\mathrm{OH}$ group (Supplementary material).

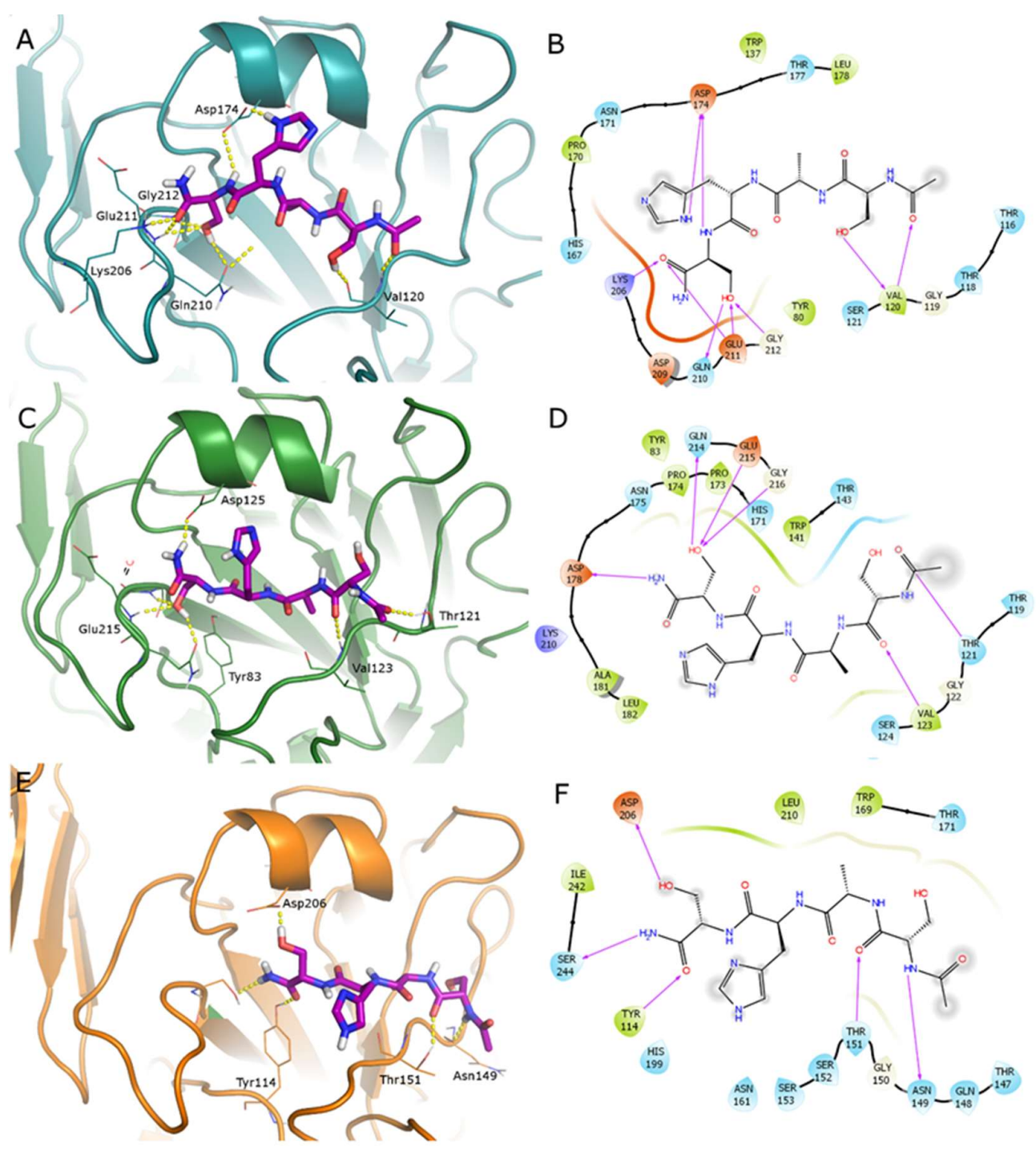

Figure 4. Docked poses of 4 (purple $C$ atoms, represented as stick) in the RBS of studied HAs represented as cartoon: A) Parma/H1N1 (deep-cyan); B) Roma/H1N1 (dark green); C) Parma/H3N2 (orange). Residues involved in $\mathrm{H}$-bond interactions with the ligand are represented as lines; H-bond are depicted as yellow dashed lines. Corresponding 2D ligand interaction diagrams are reported in panels B), D) and E); in the diagrams residues close to the ligand are coloured on the basis of their properties (orange, negatively charged; blue, positively charged; green, hydrophobic; cyan, polar), H-bonds are depicted as magenta arrows, solvent exposed atoms are surrounded by a grey shadow. 
The binding site on the stalk region of HA was derived from recently resolved X-ray complexes of H1 HA bound to small molecules [26, 27]. In both cases, the ligands occupy the HA epitope recognized by universal antibodies on the HA stem. Moreover, the same region of HA surface was found interacting with bLf in several binding poses obtained through protein-protein docking [11]. Also in this case, we checked if the same region of our model HAs could host our peptides through a SiteMap calculation. In the A/H1N1 strains, the identified site resides close to the binding epitope of crystallographic ligands, while in the H3, the site resulted very small. Therefore, for this latter HA, the TBHQ crystallographic binding site [28] was exploited for peptide docking as already done by Kim et al [29].

The analysis of obtained binding poses (Fig. 5) shows as interacting residues are common to those involved in the binding with other ligands occupying this site such as peptide fusion inhibitors [30]. Moreover, the key role of serine residues in the interactions are highlighted also in the binding of this region.

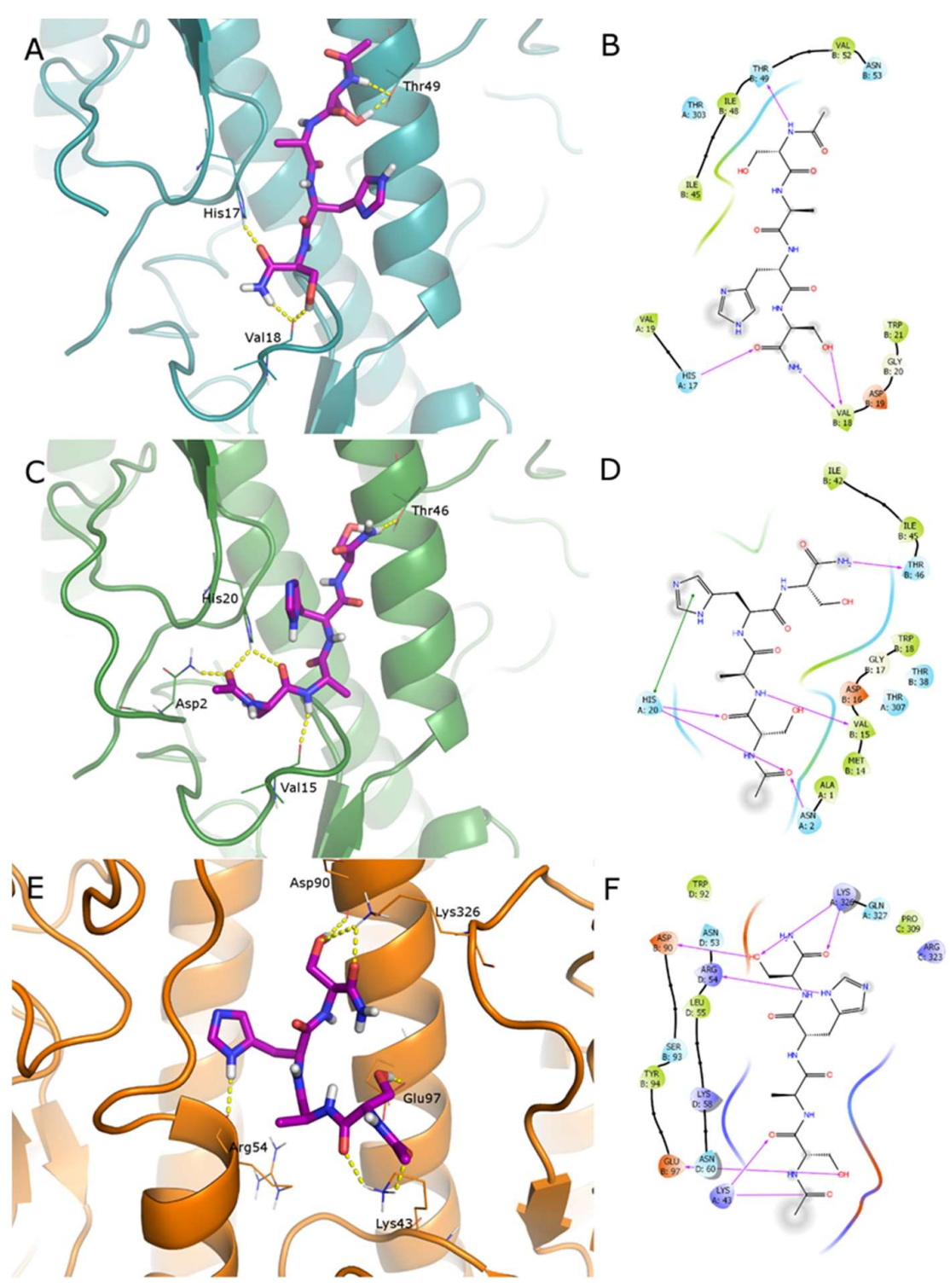

Figure 5. Docked poses of 4 (purple $\mathrm{C}$ atoms, represented as stick) in the stem sites of studied HAs represented as cartoon: A) A/Parma H1N1 (deep-cyan); B) A/Roma H1N1 
(dark green); C) A/Parma H3N2 (orange). Residues involved in H-bond interactions with the ligand are represented as lines; H-bond are depicted as yellow dashed lines. Corresponding 2D ligand interaction diagrams are reported in panels B), D) and E); in the diagrams residues close to the ligand are colored on the basis of their properties (orange, negatively charged; blue, positively charged; green, hydrophobic; cyan, polar), H-bonds are depicted as magenta arrows, solvent exposed atoms are surrounded by a grey shadow.

\section{Materials and Methods}

\subsection{Synthesis}

$\mathrm{N} \alpha$-Fmoc-protected amino acids, Rink amide-resin, coupling reagents, N, N-Diisopropylethylamine (DIEA), piperidine and trifluoroacetic acid (TFA) were purchased from Iris Biotech (Germany). Peptide synthesis solvents, reagents, as well as $\mathrm{CH}_{3} \mathrm{CN}$ for High Performance Liquid Chromatography (HPLC) were reagent grade and were acquired from commercial sources and used without further purification unless otherwise noted.

\subsubsection{Peptide synthesis}

The synthesis of tetrapeptides (1-10) was performed according to the solid phase approach using standard Fmoc (9-Fluorenylmethoxycarbonyl) methodology [31, 32] in a manual reaction vessel on a Rink amide resin $(0.150 \mathrm{~g}$, loading $0.7 \mathrm{mmol} / \mathrm{g})$ previously Fmoc-deprotected by a $25 \%$ piperidine solution in DMF $(1 \times 5 \mathrm{~min}$ and $1 \times 25 \mathrm{~min})$. Each coupling reaction was accomplished using a 3 -fold excess of amino acid with HBTU (2-(1H-benzotriazole-1-yl)-1,1,3,3-tetramethyluronium hexafluorophosphate) and $\mathrm{HOBt}$ (1-Hydroxybenzotriazole) in the presence of DIEA (6 eq.). The peptide resin was washed with dichloromethane (DCM, 3×), N,N-dimethylformamide (DMF, 3×), and DCM (3×) and the Fmoc deprotection protocol, described above, was repeated after each coupling step. After peptide assembling the N-terminal Fmoc group was removed and the peptides were acetylated adding a solution of $\mathrm{Ac}_{2} \mathrm{O} / \mathrm{DCM}$ (1:3) shaking for $30 \mathrm{~min}$. Finally, peptides were released from the resin using a cleavage mixture containing 90\% TFA, 5\% Triisopropylsilane (TIS) and $5 \% \mathrm{H}_{2} \mathrm{O}$ for $2 \mathrm{~h}$. The resin was removed by filtration, and the crude peptide was recovered by precipitation with cold anhydrous ethyl ether to give a white powder and then lyophilized.

\subsubsection{Purification and Characterization}

All crude peptides were purified by RP-HPLC on a preparative C18-bonded silica column (Phenomenex Kinetex AXIA $100 \AA$, $100 \times 21.2 \mathrm{~mm}, 5 \mu \mathrm{m}$ ) using a Shimadzu SPD 20 A UV/VIS detector, with detection at 214 and $254 \mathrm{~nm}$. Mobile phase was: (A) $\mathrm{H}_{2} \mathrm{O}$ and (B) ACN, both acidified with $0.1 \%$ TFA (v/v). Injection volume was $5000 \mu \mathrm{L}$; flow rate was set to $15 \mathrm{~mL} / \mathrm{min}$. The following gradient was employed: 0-18 $\mathrm{min}, 1-40 \% \mathrm{~B}, 18.01-20 \mathrm{~min}$, $40-70 \% \mathrm{~B}, 20.01-21 \mathrm{~min}, 70-90 \% \mathrm{~B}, 21.01-23 \mathrm{~min}$, returning to 1\% B. Analytical purity and retention time (tr) of each peptide were determined using HPLC conditions in the above solvent system (solvents $A$ and B) programmed at a flow rate of $0.800 \mathrm{ml} / \mathrm{min}$, fitted with C-18 column Supelco, Ascentis express peptide C18 column (50x3.00 mm, $2.7 \mu \mathrm{m})$. LC gradient was the following: 0-7 $\mathrm{min}, 1-40 \% \mathrm{~B}, 7.01-8 \mathrm{~min}, 40-90 \% \mathrm{~B}, 8.01-9 \mathrm{~min}$, returning to $1 \% \mathrm{~B}, 9-11 \mathrm{~min}$, isocratic for $2 \mathrm{~min}$. All analogues showed $>97 \%$ purity when monitored at $220 \mathrm{~nm}$. Homogeneous fractions, as established using analytical HPLC, were pooled and lyophilized.

Ultra high resolution mass spectra were obtained by positive ESI infusion on a LTQ Orbitrap XL mass spectrometer (Thermo Scientific, Germany), equipped with the Xcalibur 
software for processing the data acquired. The sample was dissolved in a mixture of water and methanol (50/50) and injected directly into the electrospray source, using a syringe pump, at constant flow $(15 \mu \mathrm{L} / \mathrm{min})$. The temperature of the capillary was set at $220^{\circ} \mathrm{C}$.

\subsection{Direct binding assay}

HA was purchased from Genscript, cat n. Z03181-100. CM5 sensor chips, HBS-P+ buffer (0.01 M HEPES pH 7.4, 0.15 M NaCl, 0.05 \% v/v Surfactant P20), 1-ethyl-3-(3-diaminopropyl) carbodiimide hydrochloride (EDC), N-hydroxysuccinimide (NHS), ethanolamine $\left(\mathrm{H}_{2} \mathrm{~N}\left(\mathrm{CH}_{2}\right)_{2} \mathrm{OH}\right)$, and regeneration solution were purchased from GE Healthcare.

\subsubsection{Microscale thermophoresis (MST)}

MST experiments were carried out using the Monolith NT.115pico instrument (NanoTemper Techonologies, Munich, Germany). His-HA was labeled using the Nanotemper HisTag Labeling Kit RED-tris-NTA 2nd Generation as described elsewhere [33]. Briefly, 100 $\mu \mathrm{L}$ of a solution of His-HA protein $(80 \mathrm{nM})$ in double distilled water was mixed with 100 $\mu \mathrm{L}$ of $40 \mathrm{nM}$ NT647-NHS fluorophore (NanoTemper Technologies) in labeling buffer and incubated for $30 \mathrm{~min}$ at room temperature. NT647-HA was centrifuged for $10 \mathrm{~min}$ at $4^{\circ} \mathrm{C}$ at $15000 \mathrm{~g}$ to remove protein aggregates. Pretests using standard-treated and premiumcoated MST capillaries (NanoTemper Technologies) were performed to test the adsorption of labeled HA to capillary walls by analyzing capillary scans recorded by the Monolith NT.115pico prior to MST experiments [34]. The protein did not adsorb to standardtreated capillary walls in assay buffer (PBS). For this reason, the following experiments were performed using standard-treated capillary. Then, buffer conditions were evaluated to identify optimal state for MST signal reproducibility and the suppression of unspecific adsorption to capillary walls. Compound stocks (5 mM) in MST buffer were diluted in the assay buffer to reach the highest soluble concentration $(50 \mu \mathrm{M})$. In MST experiments, 16fold 1:1 serial dilutions of each compound were mixed with NT647-HA to yield a final reaction volume of $20 \mu \mathrm{l}$. After $10 \mathrm{~min}$ of incubation at $\mathrm{rt}$, the reaction mixtures were loaded into standard-treated capillaries and subsequently inserted in the chip tray of Monolith NT.115 for thermophoresis analysis and the appraisal of $K_{D}$ values. Signals were recorded at high MST power and 10\% LED power. $K_{D}$ values were calculated from compound concentration-dependent changes in normalized fluorescence ( $\left.F_{n o r m}\right)$ of HA after 21s of thermophoresis. Each compound was tested in triplicate and data analyzed using MO Affinity Analysis software (NanoTemper Technologies, Munich, Germany). Confidence values $( \pm)$ were indicated next to $K_{D}$ value for each of tested compound.

\subsubsection{Surface Plasmon Resonance (SPR)}

SPR binding studies were performed at $25{ }^{\circ} \mathrm{C}$ using Biacore T200 (GE Health Sciences Inc.). His-HA were stably captured at the surface of the CM5 sensor chip by the means of an anti-histidine antibody (His Capture Kit, GE Healthcare) that had been covalently bound to the surface as recommended by the manufacturer. In particular, the anti-histidine antibody provided in His Capture Kit was diluted to $50 \mu \mathrm{g} / \mathrm{ml}$ in the immobilization buffer included in the kit and covalently coupled to Sensor Chip CM5 by standard amine coupling to a level of approximately $12000 \mathrm{RU}$. Then, His-HA was injected $\left(21,5 \mu \mathrm{g} \cdot \mathrm{mL}^{-1}\right.$ in $10 \mathrm{~mm}$ acetate, $\mathrm{pH} 4.59$ ) over the anti-histidine antibody surface for $1 \mathrm{~min}$. No protein was injected over the reference surface. The dissociation was monitored by injecting running buffer for $600 \mathrm{~s}$. Surface regeneration was done by injecting glycine buffer (10 mM, $\mathrm{pH} 1.5,1 \mathrm{~min})$.

HBS-P+ buffer was used as a running buffer. After the immobilization of HA, HBS-P+ buffer was injected over the chip at a flow rate of $5 \mu \mathrm{L} / \mathrm{min}$ overnight. A solution of 
peptide in HBS-P+ buffer at various concentrations (from 0.32 to $10 \mu \mathrm{M}$ ) was injected at $25^{\circ} \mathrm{C}$ with a flow rate of $30 \mu \mathrm{L} / \mathrm{min}$ for $120 \mathrm{~s}$ (association phase), and then the buffer alone was injected for $600 \mathrm{~s}$ (dissociation phase).

The equilibrium dissociation constants $\left(\mathrm{K}_{\mathrm{D}}\right)$ and kinetic dissociation $(\mathrm{kd})$ and association (ka) constants were calculated from the sensorgrams by global fitting of a 1:1 binding model using BIAevaluation software (v3.1) provided with the Biacore T200 instrument (GE Healthcare).

4.3 Biological assay

\subsubsection{Cells and viral strains.}

Madin-Darby canine kidney (MDCK, ATCC, CRL-2936) cells were grown at $37^{\circ} \mathrm{C}$ in minimal essential medium (MEM, Invitrogen, Paisley, UK) containing $1.2 \mathrm{~g} / 1 \mathrm{NaHCO}$, and supplemented with 10\% inactivated fetal calf serum (FCS, Invitrogen, Paisley, UK), 2 mM glutamine, nonessential amino acids, penicillin (100 IU/ml), and streptomycin (100 $\mu \mathrm{g} / \mathrm{ml})$. The following IAV strains were used: A/RomaISS/02/08 H1N1 (Brisbane-like) oseltamivir-sensitive virus, A/Parma/24/09 H1N1 (Brisbane-like) oseltamivir-resistant virus, and A/Parma/05/06 H3N2 (Wisconsin-like) virus. Viruses were propagated in MDCK cells in serum-free MEM supplemented with 4\% Bovine Serum Albumin (BSA fraction V, Gibco; Paisley, UK), $1 \mu \mathrm{g} / \mu \mathrm{L}$ N-tosyl-L-phenylalanine chloromethyl ketone-treated trypsin (Sigma Chemical Co.; St. Louis, MO, USA). When extensive cytopathic effect (c.p.e.) was observed, infected cultures were frozen and thawed three times, centrifuged $(3,000$ $\mathrm{rpm}, 10 \mathrm{~min}$ ), and supernatants were stored at $-80^{\circ} \mathrm{C}$. Titres of virus stocks were determined by hemagglutinin titration and/or plaque assay according to the standard procedures $[35,36]$.

\subsubsection{Cytotoxicity assay}

This procedure was performed as reported elsewhere [37]. Briefly, two-fold serial dilutions of each peptides in culture medium were incubated at $37^{\circ} \mathrm{C}$ with confluent MDCK cells grown in 96-well tissue culture microplates (Nalge Nunc Europe Ltd, Neerijse, Belgium). After 24 hours, the following parameters were evaluated: cell morphology was examined by light microscopy, cell viability was determined by neutral red staining as already described by us [38], and cell proliferation was evaluated quantitatively by microscopic counts after dispersion into individual cells with trypsin. Peptide dilutions that did not affect any of these parameters were considered as non-cytotoxic concentrations and utilized for antiviral assays.

\subsubsection{Hemagglutination inhibition assay $(\mathrm{HI})$}

Viruses in PBS (Phosphate-buffered saline, $\mathrm{pH} 7.4$ ) were incubated for 1 hour at $4^{\circ} \mathrm{C}$ with serial dilutions of peptides in PBS. An equal volume of $0.5 \%$ turkey erythrocytes was then added and allowed to agglutinate. Titres were expressed as the reciprocal of the peptide dilutions giving $50 \%$ hemagglutination of erythrocytes by four virus-agglutinating units.

\subsubsection{Neutralization assay (NT)}

NT was carried out by incubating serial two-fold peptide dilutions, starting from $12.5 \mu \mathrm{M}$, in culture medium with equal volumes of viral suspension containing $10^{6}$ plaque forming units (p.f.u.) for 1 hour at $4^{\circ} \mathrm{C}$. In negative controls, culture medium was used instead of peptides in the same volume. MDCK cells, grown in 96-well tissue culture microplates (Nalge Nunc Europe Ltd, Neerijse, Belgium), were infected with 100 l/well (10 p.f.u./cell; 
in quadruplicate) of the virus-peptide mixtures. After adsorption, cells were rinsed thoroughly and incubated at $37^{\circ} \mathrm{C}$ for 24 hours. The viral c.p.e. was measured by neutral red staining as reported elsewhere by our laboratory [37].

4.4 Computational studies

4.4.1 Homology modeling

Homology models for the three viral strains were generated using the Swiss Model webserver (https://swissmodel.expasy.org/) [39]. The fasta nucleotide sequence of the three HAs (A/Parma H1N1, A/Roma H1N1, A/Parma H3N2) was used to search for best matching proteins. To obtain the trimeric form, the quaternary structure annotation was introduced in the fasta sequence. Suitable templates were aligned using BLAST (Basic Local Alignment Search Tool) [40] and HHblits (Hidden Markov model - based lightning-fast iterative sequence search) [27]. The highest ranking template was selected to build the protein models using ProMod3 [28]. The quality of obtained models was assessed using QMEAN (Qualitative Model Energy ANalysis) [41] and GMQE (Global Model Quality Estimate). The Ramachandran Plot for each obtained structure was generated to check stereochemical and conformational quality. The validity of obtained quaternary structure was provided by the QSQE (quaternary structure quality estimate) score [42]. Identified templates and related score values for the three models are reported in the Supplementary material.

\subsubsection{Protein Preparation}

The three proteins were aligned in Maestro using the Protein structure alignment tool that performs the process based on the sequence alignment on a reference protein, A/Roma $\mathrm{H} 1 \mathrm{~N} 1$ in our case.

Aligned homology models were submitted to the Protein Preparation routine in Maestro in order to optimize the obtained structures. In particular, the H-bond optimizer and the restrained minimization were carried out to ameliorate the H-bond network.

\subsubsection{Binding site identification and analysis}

To locate the putative binding site on the receptor binding domain of the studied HAs, the homology models were aligned to the available X-ray complex of pandemic HA bound to 6'-SLN (PDB ID: 3UBN [25]). The binding site on the stem region was derived from the ligand position identified in 6CF7 [26] and 6WCR [43] for H1N1; while, the X-ray complex of H3 hemagglutinin with THBQ (PDB ID: 3EYK, 3EYM [43]) was used to locate the binding site on the Parma/H3N2.

Each putative site was explored performing a SiteMap [21, 45] calculation using the following settings: evaluate a single binding site using the aligned X-ray ligand as a reference. The fine grid and more restrictive definition of hydrophobicity was applied. The site was cropped at $6 \AA$ from the last sitepoint.

\subsubsection{Receptor grid generation}

For all the sites mentioned in the previous paragraph, the receptor grid was generated using the Receptor Grid Generation routine available in Glide [22, 23, 24, 45]. The grid box was enlarged to dock ligands $<=20 \AA$ and the inner box size was set to: $x 10, y 15, z 10$. All grids were generated with settings suitable to peptide docking. 
All sites in the RBD and the one corresponding the TBHQ binding region were located exploiting the SiteMap calculated sitepoints The center of sites on the stem region of H1N1 strains was defined as the centroid of selected residues (His17, Ile45, Ile48 for A/Parma H1N1 and His20, Ile42, Ile45 for A/Roma H1N1).

In the RBS the following rotatable groups were set: Thr147, Thr151, Ser152, Ser153, Thr171, Thr203, Ser209 for Parma/H3N2; Tyr80, Ser122, Thr177, Tyr179 for A/Parma H1N1 and Tyr83, Thr121, Se133, Thr143, Tyr183 for Roma/H1N1. Ser93 and Tyr94 were defines as rotatable groups in the TBHQ binding site (A/Parma H3N2); while in the stem binding sites Thr16, 41, 49, Thr303 were set rotatable for A/Parma H1N1 and Thr19, 38, 46, Thr307 for Roma/H1N1.

\subsubsection{Ligand preparation}

The structures of the studied peptides 1-10 were built in Maestro using the Build tool. Obtained structures were submitted to Ligprep to generate possible tautomers and protomers at physiological $\mathrm{pH}(7.0 \pm 0.4)$. Resulting structures were minimized to a derivative convergence of $0.001 \mathrm{~kJ} / \mathrm{mol}-1$ using the PRCG minimization algorithm, the OPLS3e force field, and the generalized Born/surface area (GB/SA) water solvation model implemented in MacroModel [45].

\subsubsection{Docking calculations}

Minimized ligand structures were docked in all the previously identified sites on the HA surface using Glide and the SP-peptide docking protocol that allows to increase the conformational exploration of ligands affording a final set of 100 poses. Obtained poses for each ligand were clustered using the Clustering of conformers tool available in Maestro. The Average clustering algorithm was used on the basis of the RMSD calculated between heavy atom pairs. The Kelley penalty was calculated and the optimal clustering level was set consequently. Most populated clusters were evaluated along with the docking score.

\section{Conclusions}

In this paper, we performed a systematic SAR study through the development of alascan peptides starting from the most promising tetrapeptides identified previously [13]. Direct binding assay were carried out exploiting two biophysical methods (MST and SPR), while the evaluation of antiviral activity was assessed through both HI and NT studies. The structure-based computational studies allowed to envision the putative interactions of this ligand with HA, highlighting the role of the serine residues in the receptor binding. All applied methods agreed upon the identification of a novel potent tetrapeptide, SAHS, able to bind hemagglutinin with high affinity and inhibit the Influenza virus hemagglutination and cell infection at femtomolar concentration. This small sequence, with high and broad-spectrum activity, can represent a valuable starting point for the design of small molecules. The work carried out, in fact, opens the way to new perspectives for the development of new anti-Influenza drugs especially in a context in which the emergence of new and drug-resistant viruses highlights the need for new antiviral approaches and strategies.

Supplementary Materials: The following are available online at www.mdpi.com/xxx/s1.

Author Contributions: M.S. and P.C: supervision. M.S.: conceptualization and project administration. M.S. and M.C.S.: biophysical assay. M.S., M.C.S., M.A., F.S.: data curation, writing-original draft preparation, formal analysis. M.C.S. and V.D.: methodology, synthesis. M.A.: Computational studies. M.S., M.A., F.S., P.C.: writing-review. A.P. and F.S.: biological assay. A.B.: resources. P.C.: 
funding acquisition and editing. All authors have read and agreed to the published version of the manuscript.

Funding: This work was supported by Grants from the National Institute of Health "Studi sull'attività antimicrobica di sostanze di origine naturale o sintetiche" (F.S.).

Institutional Review Board Statement: The study did not involve humans or animals.

Informed Consent Statement: The study did not involve humans.

Data Availability Statement: The data presented in this study are available on request from the corresponding author.

Acknowledgments: The assistance of the staff is gratefully appreciated.

Conflicts of Interest: The authors declare no conflict of interest.

\section{References}

1. Hale, B. G.; Albrecht, R. A.; García-Sastre, A. Innate immune evasion strategies of influenza viruses. Future Microbiol. 2010, 5(1), 23-41. https://doi.org/10.2217/fmb.09.108

2. Shi, W. and Gao, G. F. Emerging H5N8 avian influenza viruses. Science (New York, N.Y.) 2021, 372(6544), 784-786. https://doi.org/10.1126/science.abg6302

3. Hayden F. G. Respiratory viral threats. Curr. Opin. Infect. Dis. 2006, 19(2), 169-178. https://doi.org/10.1097/01.qco.0000216628.51563.b1

4. de St Maurice, A.; Halasa, N. Preparing for the 2019-2020 influenza season. Pediatr. Transplant. 2020, 24(1), e13645. https://doi.org/10.1111/petr.13645

5. Alves Galvão, M. G.; Rocha Crispino Santos, M. A.; Alves da Cunha, A. J. Amantadine and rimantadine for influenza A in children and the elderly. Cochrane Database Syst. Rev. 2014, 11, CD002745. https://doi.org/10.1002/14651858.

6. Kamali, A.; Holodniy, M. Influenza treatment and prophylaxis with neuraminidase inhibitors: a review. Infect. Drug. Resist. 2013, 6, 187-198. https://doi.org/10.2147/IDR.S36601

7. Shie, J. J.; Fang, J. M. Development of effective anti-influenza drugs: congeners and conjugates - a review. J Biomed Sci. 2019, 26(1):84. https://doi: 10.1186/s12929-019-0567-0.

8. Aschenbrenner D. S. Xofluza Now Indicated to Prevent Influenza. Am. J. Nurs. 2021, 121(2):26-27. https://doi.org/10.1097/01.NAJ.0000734116.32090.78

9. Belshe, R. B.; Burk, B.; Newman, F.; Cerruti, R. L.; Sim, I. S. Resistance of influenza A virus to amantadine and rimantadine: results of one decade of surveillance. J. Infect. Dis. 1989, 159(3), 430-435. https://doi.org/10.1093/infdis/159.3.430

10. Bertram, S.; Glowacka, I.; Steffen, I.; Kühl, A.; Pöhlmann, S. Novel insights into proteolytic cleavage of influenza virus hemagglutinin. Rev. Med. Virol. 2010, 20(5), 298-310. https://doi.org/10.1002/rmv.657.

11. Ammendolia, M. G.; Agamennone, M.; Pietrantoni, A.; Lannutti, F.; Siciliano, R. A.; De Giulio, B.; Amici, C.; Superti, F. Bovine lactoferrin-derived peptides as novel broad-spectrum inhibitors of influenza virus. Pathog. Glob. Health. 2012, 106(1), 12-19. https://doi.org/10.1179/2047773212Y.0000000004

12. Superti, F.; Agamennone, M., Pietrantoni, A., Ammendolia, M. G. Bovine Lactoferrin Prevents Influenza A Virus Infection by Interfering with the Fusogenic Function of Viral Hemagglutinin. Viruses 2019, 11(1), 51. https://doi.org/10.3390/v11010051

13. Scala, M. C.; Sala, M.; Pietrantoni, A.; Spensiero, A.; Di Micco, S.; Agamennone, M.; Bertamino, A.; Novellino, E.; Bifulco, G.; Gomez-Monterrey, I. M.; Superti, F.; Campiglia, P. Lactoferrin-derived Peptides Active towards Influenza: Identification of Three Potent Tetrapeptide Inhibitors. Sci. Rep. 2017, 7(1), 10593. https://doi.org/10.1038/s41598-017-10492-x

14. Gomez-Monterrey, I.; Sala, M.; Rusciano, M. R.; Monaco, S.; Maione, A. S.; Iaccarino, G.; Tortorella, P.; D'Ursi, A. M.; Scrima, M.; Carotenuto, A.; De Rosa, G.; Bertamino, A.; Vernieri, E.; Grieco, P.; Novellino, E.; Illario, M.; Campiglia, P. 
Characterization of a selective CaMKII peptide inhibitor. Eur. J. Med. Chem. 2013, 62, 425-434. https://doi.org/10.1016/j.ejmech.2012.12.053

15. Renaud, J. P.; Chung, C. W.; Danielson, U. H.; Egner, U.; Hennig, M.; Hubbard, R. E.; Nar, H. Biophysics in drug discovery: impact, challenges and opportunities. Nat. Rev. Drug. Discov. 2016, 15(10), 679-698. https://doi.org/10.1038/nrd.2016.123

16. Duhr, S.; Braun, D. Why molecules move along a temperature gradient. PNAS 2006, 103(52), 19678-19682. https://doi.org/10.1073/pnas.0603873103

17. Navratilova, I.; Hopkins, A. L. Fragment screening by surface plasmon resonance. ACS med. chem. lett. 2010, 1(1), 44-48. https://doi.org/10.1021/m1900002k

18. Kadam, R. U.; Wilson, I. A. A small-molecule fragment that emulates binding of receptor and broadly neutralizing antibodies to influenza A hemagglutinin. PNAS 2018, 115(16), 4240-4245. https://doi.org/10.1073/pnas.1801999115

19. Yusuf, M.; Konc, J.; Sy Bing, C.; Trykowska Konc, J.; Ahmad Khairudin, N. B.; Janezic, D.; Wahab, H. A. Structurally conserved binding sites of hemagglutinin as targets for influenza drug and vaccine development. J. Chem. Inf. Model. 2013, 53(9), 2423-2436. https://doi.org/10.1021/ci400421e

20. Weis, W.; Brown, J. H.; Cusack, S.; Paulson, J. C.; Skehel, J. J.; Wiley, D. C. Structure of the influenza virus haemagglutinin complexed with its receptor, sialic acid. Nature 1988, 333(6172), 426-431. https://doi.org/10.1038/333426a0

21. Halgren T. A. Identifying and characterizing binding sites and assessing druggability. J. Chem. Inf. Model. 2009, 49(2), 377389. https://doi.org/10.1021/ci800324m

22. Friesner, R. A.; Banks, J. L.; Murphy, R. B.; Halgren, T. A.; Klicic, J. J.; Mainz, D. T.; Repasky, M. P.; Knoll, E. H.; Shelley, M.; Perry, J. K.; Shaw, D. E.; Francis, P.; Shenkin, P. S. Glide: a new approach for rapid, accurate docking and scoring. 1. Method and assessment of docking accuracy. J. Med. Chem. 2004, 47(7), 1739-1749. https://doi.org/10.1021/jm0306430

23. Halgren, T. A.; Murphy, R. B.; Friesner, R. A.; Beard, H. S.; Frye, L. L.; Pollard, W. T.; Banks, J. L. Glide: a new approach for rapid, accurate docking and scoring. 2. Enrichment factors in database screening. J. Med. Chem. 2004, 47(7), 1750-1759. https://doi.org/10.1021/jm030644s

24. Friesner, R. A.; Murphy, R. B.; Repasky, M. P.; Frye, L. L., Greenwood, J. R.; Halgren, T. A.; Sanschagrin, P. C.; Mainz, D. T. Extra precision glide: docking and scoring incorporating a model of hydrophobic enclosure for protein-ligand complexes. J. Med. Chem. 2006, 49(21), 6177-6196. https://doi.org/10.1021/jm051256o

25. Xu, R.; McBride, R.; Nycholat, C. M.; Paulson, J. C.; Wilson, I. A. Structural characterization of the hemagglutinin receptor specificity from the 2009 H1N1 influenza pandemic. J Virol. 2012, 86(2), 982-990. https://doi.org/10.1128/JVI.06322-11

26. van Dongen, M.; Kadam, R. U.; Juraszek, J.; Lawson, E.; Brandenburg, B.; Schmitz, F.; Schepens, W.; Stoops, B.; van Diepen, H. A.; Jongeneelen, M.; Tang, C.; Vermond, J.; van Eijgen-Obregoso Real, A.; Blokland, S.; Garg, D.; Yu, W.; Goutier, W.; Lanckacker, E.; Klap, J. M.; Peeters, D.; Wu, J.; Buyck, C.; Jonckers, T.H.M.; Roymans, D.; Roevens, P.; Vogels, R.; Koudstaal, W.; Friesen, R.H.E.; Raboisson, P.; Dhanak, D.; Goudsmit, J.; Wilson, I. A. A small-molecule fusion inhibitor of influenza virus is orally active in mice. Science 2019, 363(6431), eaar6221. https://doi.org/10.1126/science.aar6221

27. Remmert, M.; Biegert, A.; Hauser, A.; Söding, J. HHblits: lightning-fast iterative protein sequence searching by HMM-HMM alignment. Nat. methods 2012, 9(2), 173-175. https://doi.org/10.1038/nmeth.1818

28. Biasini, M.; Bienert, S.; Waterhouse, A.; Arnold, K.; Studer, G.; Schmidt, T.; Kiefer, F.; Gallo Cassarino, T.; Bertoni, M.; Bordoli, L.; Schwede, T. SWISS-MODEL: modelling protein tertiary and quaternary structure using evolutionary information. Nucleic Acids Res. 2014, 42, W252-W258. https://doi.org/10.1093/nar/gku340

29. Kim, J. I.; Lee, S.; Lee, G. Y.; Park, S.; Bae, J. Y.; Heo, J.; Kim, H. Y.; Woo, S. H.; Lee, H. U.; Ahn, C. A.; Bang, H. J.; Ju, H. S.; Ok, K.; Byun, Y.; Cho, D. J.; Shin, J. S.; Kim, D. Y.; Park, M. S.; Park, M. S. Novel Small Molecule Targeting the Hemagglutinin Stalk of Influenza Viruses. J Virol. 2019, 93(17), e00878-19. https://doi.org/10.1128/JVI.00878-19

30. Kadam, R. U.; Juraszek, J.; Brandenburg, B.; Buyck, C.; Schepens, W.; Kesteleyn, B.; Stoops, B.; Vreeken, R. J.; Vermond, J.; 
Goutier, W.; Tang, C.; Vogels, R.; Friesen, R.; Goudsmit, J.; van Dongen, M.; Wilson, I. A. Potent peptidic fusion inhibitors of influenza virus. Science 2017, 358(6362), 496-502. https://doi.org/10.1126/science.aan0516

31. Atherton, E. \& Sheppard, R. C. Solid-Phase Peptide Synthesis: A Practical Approach, Oxford, U.K., 1989.

32. Pescina, S.; Sala, M.; Padula, C.; Scala, M. C.; Spensiero, A.; Belletti, S.; Gatti, R.; Novellino, E.; Campiglia, P.; Santi, P.; Nicoli, S.; Ostacolo, C. Design and Synthesis of New Cell Penetrating Peptides: Diffusion and Distribution Inside the Cornea. Mol. Pharm. 2016, 13(11), 3876-3883. https://doi.org/10.1021/acs.molpharmaceut.6b00658

33. Monolith NT Protein Labeling Kit RED-NHS. User Manual.

34. Milite, C.; Feoli, A.; Horton, J. R.; Rescigno, D.; Cipriano, A.; Pisapia, V.; Viviano, M.; Pepe, G.; Amendola, G.; Novellino, E.; Cosconati, S.; Cheng, X.; Castellano, S.; Sbardella, G. Discovery of a Novel Chemotype of Histone Lysine Methyltransferase EHMT1/2 (GLP/G9a) Inhibitors: Rational Design, Synthesis, Biological Evaluation, and Co-crystal Structure. J. Med.Chem., 2019, 62(5), 2666-2689. https://doi.org/10.1021/acs.jmedchem.8b02008

35. Gaush, C. R.; Smith, T. F. Replication and plaque assay of influenza virus in an established line of canine kidney cells. Appl. Microbiol. 1968, 16(4), 588-594. https://doi.org/10.1128/am.16.4.588-594.1968

36. Rimmelzwaan, G. F.; Baars, M.; Claas, E. C.; Osterhaus, A. D. Comparison of RNA hybridization, hemagglutination assay, titration of infectious virus and immunofluorescence as methods for monitoring influenza virus replication in vitro. J. Virol. Methods. 1998, 74(1), 57-66. https://doi.org/10.1016/s0166-0934(98)00071-8

37. Pietrantoni, A.; Dofrelli, E.; Tinari, A.; Ammendolia, M. G.; Puzelli, S.; Fabiani, C.; Donatelli, I.; Superti, F. Bovine lactoferrin inhibits influenza A virus induced programmed cell death in vitro. Biometals: Biometals. 2010, 23(3), 465-475. https://doi.org/10.1007/s10534-010-9323-3

38. Marchetti, M.; Trybala, E.; Superti, F.; Johansson, M.; Bergström, T. Inhibition of herpes simplex virus infection by lactoferrin is dependent on interference with the virus binding to glycosaminoglycans. Virology 2004, 318(1), 405-413. https://doi.org/10.1016/j.virol.2003.09.029

39. Waterhouse, A.; Bertoni, M.; Bienert, S.; Studer, G.; Tauriello, G.; Gumienny, R.; Heer, F. T.; de Beer, T.; Rempfer, C.; Bordoli, L.; Lepore, R.; Schwede, T. SWISS-MODEL: homology modelling of protein structures and complexes. Nucleic Acids Res. 2018, 46(W1), W296-W303. https://doi.org/10.1093/nar/gky427

40. Camacho, C.; Coulouris, G.; Avagyan, V.; Ma, N.; Papadopoulos, J.; Bealer, K.; Madden, T. L. BLAST+: architecture and applications. BMC bioinformatics 2009, 10, 421. https://doi.org/10.1186/1471-2105-10-421

41. Benkert, P.; Biasini, M.; Schwede, T. Toward the estimation of the absolute quality of individual protein structure models. Bioinformatics 2011, 27(3), 343-350. https://doi.org/10.1093/bioinformatics/btq662

42. Bertoni, M.; Kiefer, F.; Biasini, M.; Bordoli, L.; Schwede, T. Modeling protein quaternary structure of homo- and heterooligomers beyond binary interactions by homology. Sci. Rep. 2017, 7(1), 10480. https://doi.org/10.1038/s41598-017-09654-8

43. Yao, Y.; Kadam, R. U.; Lee, C. D.; Woehl, J. L.; Wu, N. C.; Zhu, X.; Kitamura, S.; Wilson, I. A.; Wolan, D. W. An influenza A hemagglutinin small-molecule fusion inhibitor identified by a new high-throughput fluorescence polarization screen. PNAS 2020, 117(31),18431-18438. https://doi.org/10.1073/pnas.2006893117

44. Russell, R. J.; Kerry, P. S.; Stevens, D. J.; Steinhauer, D. A.; Martin, S. R.; Gamblin, S. J.; Skehel, J. J. Structure of influenza hemagglutinin in complex with an inhibitor of membrane fusion. PNAS 2008, 105(46), 17736-17741. https://doi.org/10.1073/pnas.0807142105

45. Schrödinger Release, 2020-4: Maestro, Glide, LigPrep, MacroModel, SiteMap, New York, NY, 2020. 